\title{
Severe Tricuspid Valve Regurgitation Requiring Surgical Intervention as a Result of Pacemaker Lead Extraction: A Case Series
}

\author{
BRIJESH PATEL, DO, AHMAD DARAGHMEH, MD and CHRISTIAN MACHADO, MD \\ Division of Cardiology, Providence Heart Institute, Providence Hospital and Medical Centers, Southfield, MI
}

\begin{abstract}
Tricuspid regurgitation (TR) due to pacemaker and implantable cardioverterdefibrillator (ICD) leads is a well-known condition. As these leads remain implanted, they tend to stimulate an inflammatory response leading to fibrosis and adhesions between valve tissues and the metallic component of the leads. With growing numbers of lead implantations, lead extraction procedures are also rising. The extraction of a chronic lead poses a challenge for the operators because of fibrosis and adhesions. TR after pacemaker lead extractions requiring surgical intervention is an uncommon presentation. We report two cases of severe TR after pacemaker lead extractions requiring tricuspid valve replacement.
\end{abstract}

KEYWORDS. heart failure, laser sheath, lead extraction, tricuspid regurgitation.
ISSN 2156-3977 (print) ISSN 2156-3993 (online)

(C) 2014 Innovations in Cardiac Rhythm Management

\section{Introduction}

Multiple ventricular lead placements may interfere with appropriate tricuspid valve closure during systole and may distort the tricuspid valve apparatus geometry leading to malcoaptation. This will eventually leads to tricuspid regurgitation (TR). As the pacemaker or implantable cardioverter-defibrillator (ICD) leads remain implanted for a long time, fibrosis and adhesions around the lead ensue. This poses a challenge for the operator when lead extraction is attempted, and it requires specialized tools to extract the lead. Although it is not uncommon to observe TR after lead extractions, severe complications from TR requiring surgery are rare. Herein, we report two cases of severe TR after lead extractions requiring surgical evaluations.

The authors report no conflicts of interest for the published content. Manuscript received November 19, 2013, final version accepted January 20, 2014.

Address correspondence to: Christian E. Machado, MD, Providence Heart Institute, Providence Hospital and Medical Centers, 16001 West Nine Mile Road, Southfield, MI 48075. E-mail: cmachado@ comcast.net

\section{Case 1}

A 64-year-old man presented to the hospital for removal of a failed pacemaker lead. Removal of the lead with a laser sheath and replacement was uneventful. When the extracted lead was examined, fibrosed tissue was attached to the terminal portion of the lead (Figure 1). The patient tolerated the procedure well. Two months later, he presented with a 40-pound weight gain, and had developed massive edema of the lower extremities. The physical examination was significant for jugular venous distention, Grade II/VI holosystolic murmur at the left sternal border, and lower extremity +2 edema. An echocardiogram (Figure 2) showed severe TR with signs of volume overload. A consultation with a cardiothoracic surgeon was obtained, and it was decided to proceed with tricuspid valve replacement. The gross pathologic examination of the native tricuspid valve showed a fragmented leaflet of the valve and intact chordae tendinae. Microscopic examination showed fibromyxoid valvulopathy.

\section{Case 2}

A 52-year-old man was referred to electrophysiology services for evaluation of lead fracture of a pacemaker. 


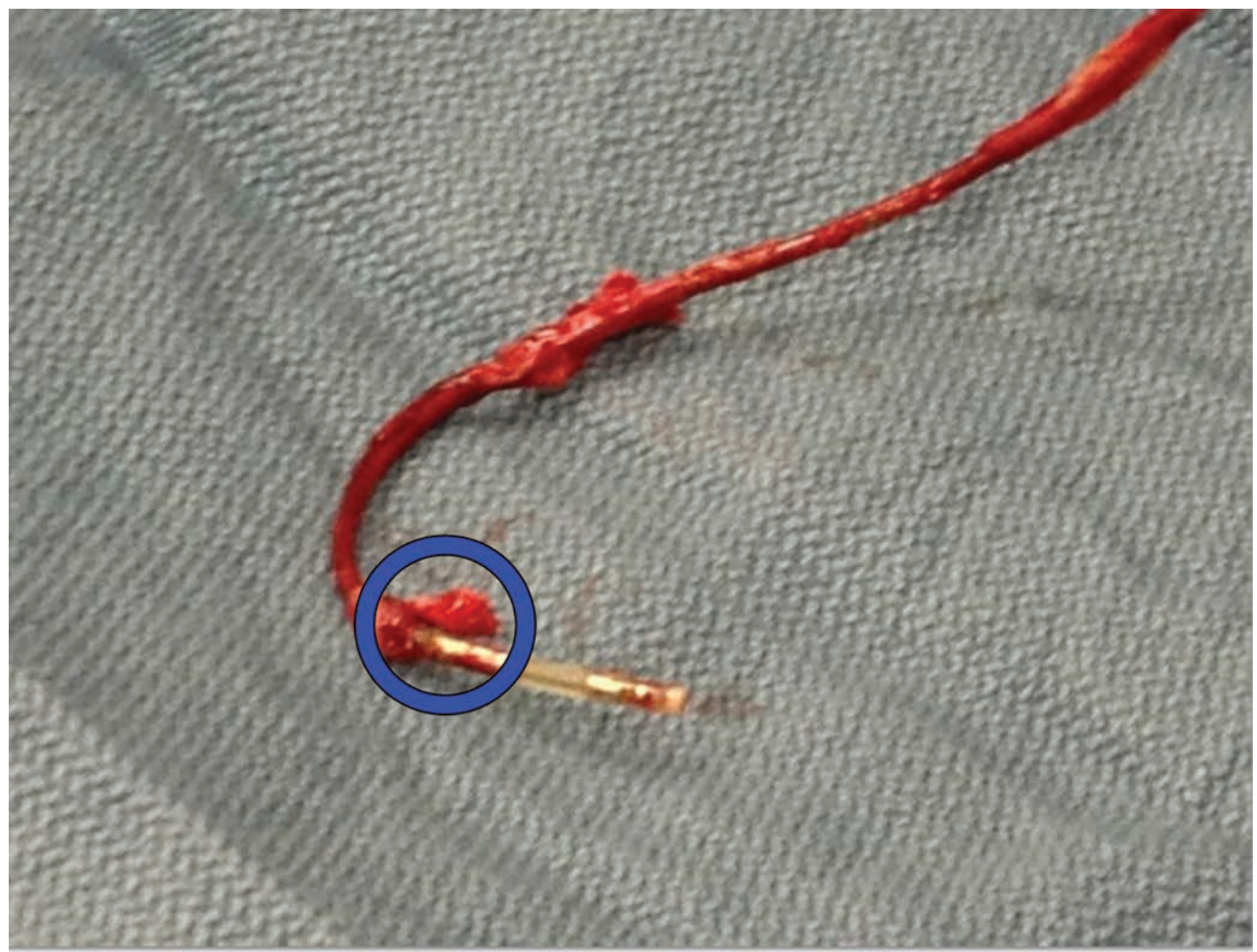

Figure 1: The failed, extracted lead shows evidence of a fibrosis attached to it (blue circle).

He had been implanted 10 years earlier for recurrent malignant syncope. The device interrogation confirmed diagnosis of a fractured lead with loss of capture and high impedance. The lead was successfully extracted with a laser sheath. On examination of the lead, cardiac tissue was attached (Figure 3) to the terminal portion of the lead. There were no procedure-related complications. He was discharged the next day. Three weeks later, he presented with right-side heart failure symptoms and dyspnea on minimal exertion. A transesophageal echocardiogram (TEE) (Figure 4) demonstrated severe TR and enlargement of the right ventricle. A cardiothoracic consultation was obtained, and the patient was advised to undergo tricuspid valve replacement surgery. At that time, the patient did not want to undergo any intervention. The patient was managed medically.

\section{Discussion}

TR is a well-known complication of implantation of pacemaker and ICD leads. The prevalence of TR in patients with pacemaker ranges from $25 \%$ to $29 \% .{ }^{1}$ Lead extraction can be a daunting task in patients with chronically implanted leads. When the leads remain implanted for a long time, fibrotic changes around the leads and abutting structures take place. The fibrosis may damage major vascular and cardiac structures when lead extraction is attempted. A case of damage to papillary muscles and chordate tendinae after lead extraction that resulted in TR has been reported. While TR due to damaged tricuspid valve apparatus is unlikely, TR is not an uncommon complication after lead extraction. The incidence of TR after lead extraction is about $9 \%{ }^{2}$ Usually, TR is mild to moderate after lead extraction; the presentation of severe TR requiring surgical intervention is rare. ${ }^{3,4}$

More than 10,000-15,000 pacemaker and ICD lead extractions are performed worldwide annually. ${ }^{5}$ The increase in the number of lead implantations will eventually result in lead extractions. Currently, infection is the most common cause for lead extraction. The risk factors that could predispose patients to develop TR after lead extractions are female gender, the use of a laser sheath and additional tools other than simple traction, the extraction of more than two leads, and the 


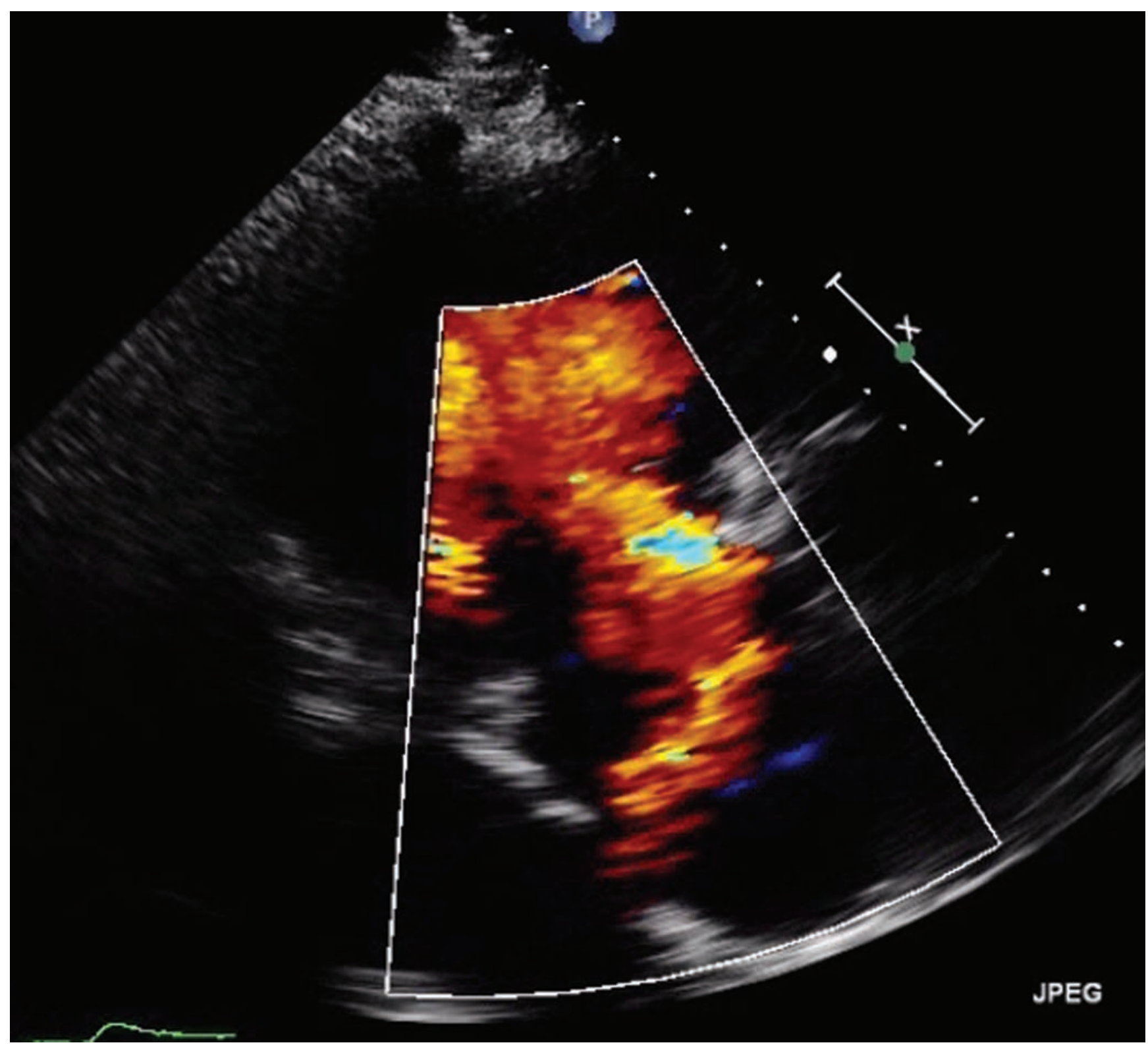

Figure 2: Color flow Doppler echocardiogram from right ventricle inflow window demonstrating a significant blood flow turbulence across the TV in systole. Further Doppler evaluation of this jet confirmed severe tricuspid regurgitation.

chronicity of lead implantation. ${ }^{2}$ Interestingly, it was observed that there was a trend towards increased mortality in the traumatic TR group, though this was not statistically significant. ${ }^{2}$ In regards to laser sheath use, conflicting data exist. Rodriguez et $\mathrm{al}^{6}$ reported that laser lead extraction was not associated with development of new tricuspid insufficiency. The laser sheath technique is reserved for patients in whom simple traction does not work, which is an indication of significant fibrosis. $^{2}$

The term lead extraction applies to a procedure that involves removal of a lead that has been implanted for more than 1 year requiring a special procedure and technology. ${ }^{7}$ In general, lead extraction is a relatively safe procedure, with perioperative mortality between $0.4 \%$ and $0.8 \%{ }^{3}$ Regardless of what technique is used to remove a lead, there are inherent risks associated with lead extraction. These include bleeding, laceration or perforation of major venous and cardiac structures, hemopericardium, infection, tricuspid valve injury, pulmonary embolism, and fatal arrhythmias. ${ }^{3}$

Intraoperative monitoring with TEE is of particular importance. It allows an operator to monitor injury to the tricuspid valve, papillary muscles, and tendinous cords; partial muscle rupture could progress to complete rupture. $^{3}$ Depending upon the severity and type of injury, patients may develop right-sided heart failure. The initial management step includes symptomatic relief with diuretics. Patients with severe heart failure due to severe TR in a structurally abnormal tricuspid valve may benefit from surgical intervention. A success rate of $85 \%$ is reported for tricuspid valve repair or replacement. ${ }^{1}$ 


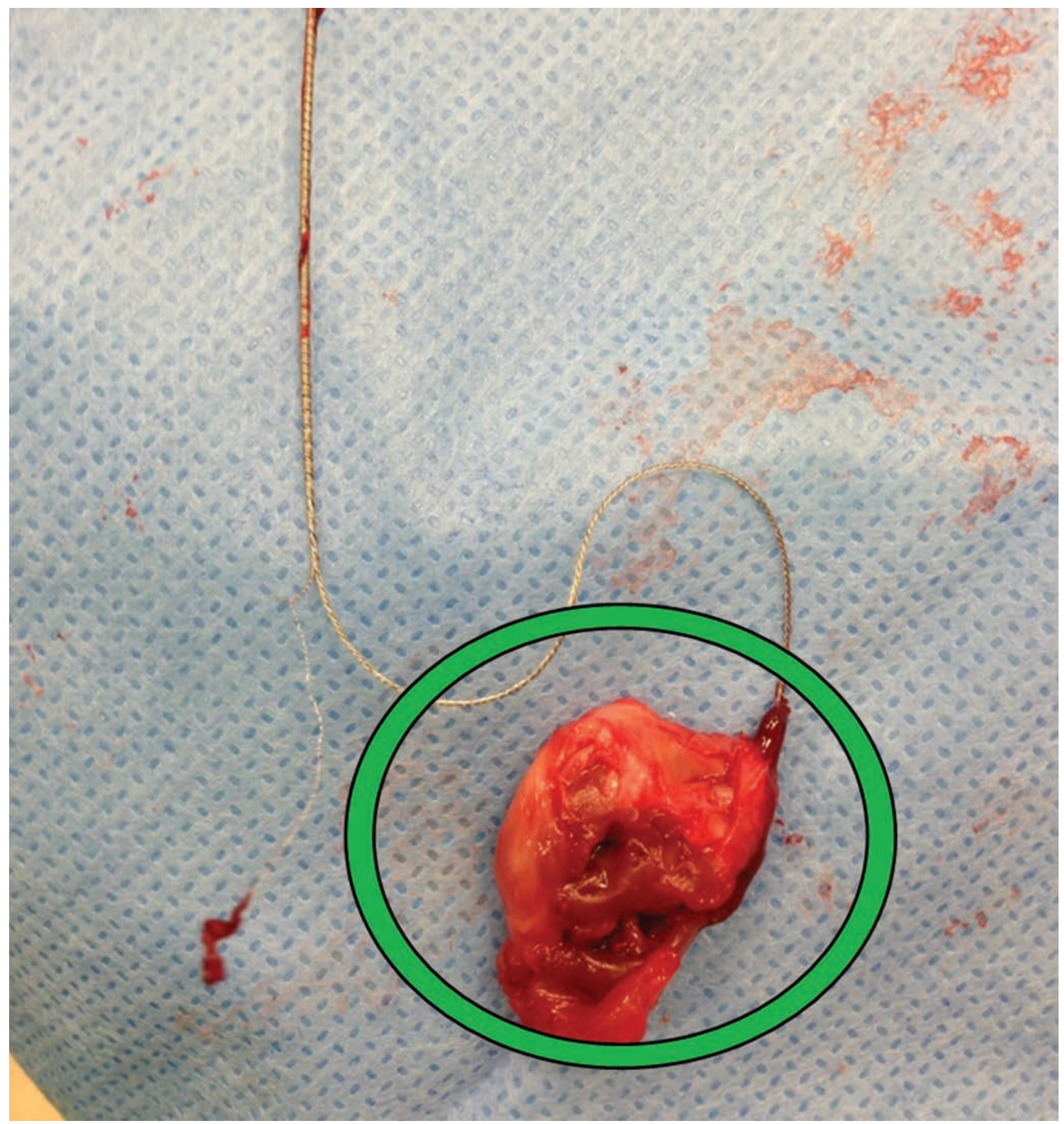

Figure 3: The adherence of cardiac tissue to the terminal portion of the extracted lead (green circle). 


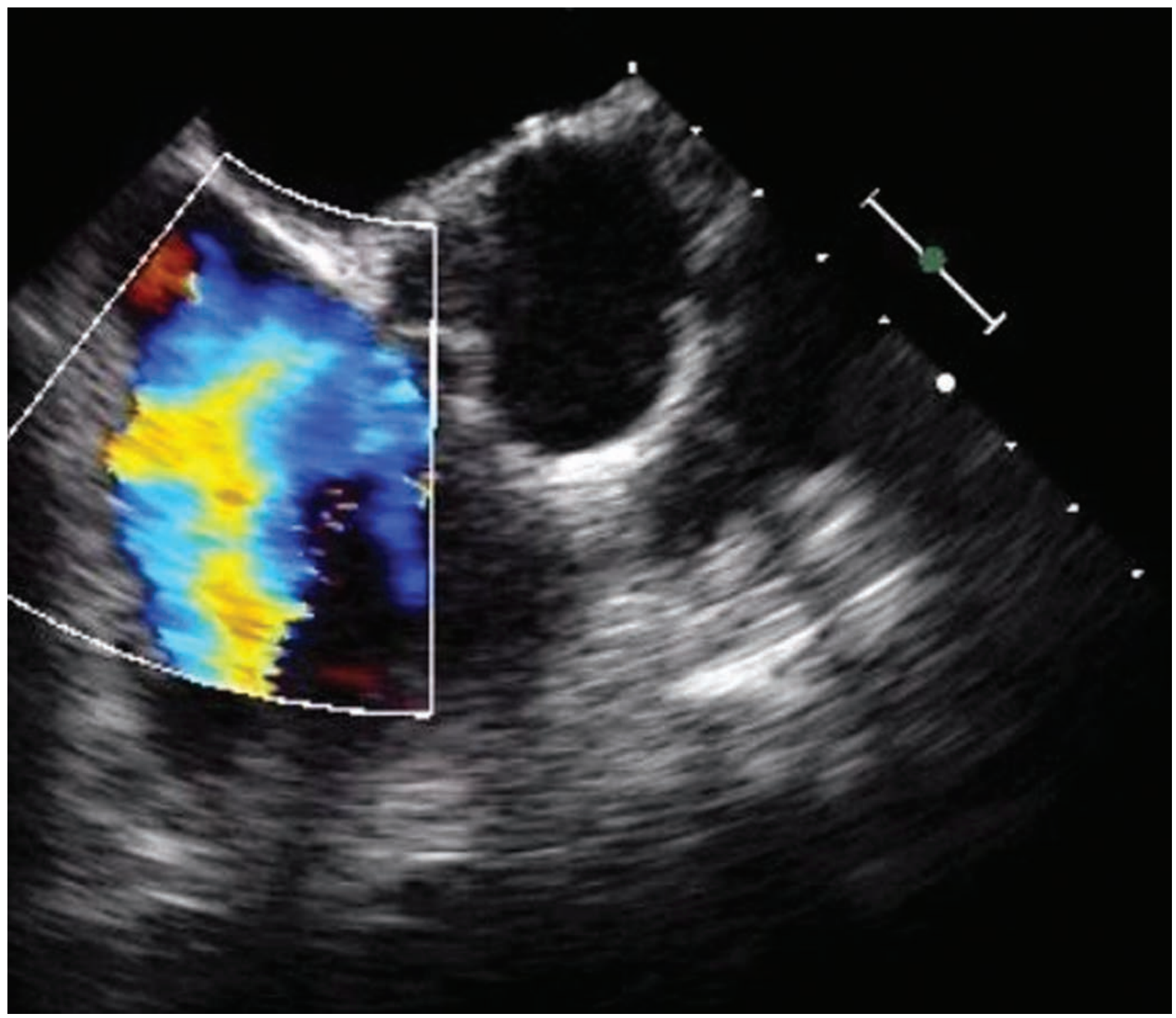

Figure 4: A transesophageal echocardiogram showing evidence of severe tricuspid regurgitation.

This makes the surgical approach a possible option for patients with severe heart failure.

\section{Conclusion}

TR from lead implantation or extraction is not uncommon, though severe TR is rare. During the extraction of a lead, fibrosis plays an important role in the development of severe TR. Severely symptomatic patients, who fail medical management, may benefit from tricuspid valve repair or replacement.

\section{References}

1. Al-Bawardy R, Krishnaswamy A, Bhargava M, et al. Clin Cardiol 2013; 36:249-254.
2. Franceschi F, Thuny F, Giorgi R, et al. J Am Coll Cardiol 2009; 53:2168-2174.

3. Mehrotra D, Kejriwal NK. Tricuspid valve repair for torrential tricuspid regurgitation after permanent pacemaker lead extraction. Tex Heart Inst J 2011; 38:305-307.

4. Roux JF, Page P, Dubuc M, et al. Pacing Clin Electrophysiol 2007; 30:214-220.

5. Hauser RG, Katsiyiannis WT, Gornick CC, Almquist AK, Kallinen LM: Deaths and cardiovascular injuries due to device-assisted implantable cardioverter-defibrillator and pacemaker lead extraction. Europace 2010; 12:395-401.

6. Rodriguez Y, Mesa J, Arguelles E, Carrillo RG. Tricuspid insufficiency after laser lead extraction. Pacing Clin Electrophysiol 2013; 36(8):939-44.

7. Wilkoff BL, Love CJ, Byrd CL, et al. Transvenous lead extraction: Heart Rhythm Society expert consensus on facilities, training, indications, and patient management: this document was endorsed by the American Heart Association (AHA). Heart Rhythm 2009; 6:1085-1104. 